\title{
Overexpression of ADAM9 in oral squamous cell carcinoma
}

\author{
PATTARAMON TANASUBSINN ${ }^{1}$, WIN PA PA AUNG ${ }^{1}$, SUPANSA PATA $^{2,3}$, \\ WITIDA LAOPAJON $^{3}$, ANUPONG MAKEUDOM ${ }^{1}$, THANAPAT SASTRARUJI ${ }^{1}$, \\ WATCHARA KASINRERK $^{2,3}$ and SUTTICHAI KRISANAPRAKORNKIT ${ }^{1}$ \\ ${ }^{1}$ Center of Excellence in Oral and Maxillofacial Biology, Department of Oral Biology and \\ Diagnostic Sciences, Faculty of Dentistry; ${ }^{2}$ Division of Clinical Immunology, Department of Medical Technology, \\ Faculty of Associated Medical Sciences; ${ }^{3}$ Biomedical Technology Research Center, National Center for \\ Genetic Engineering and Biotechnology, National Sciences and Technology Development Agency at \\ The Faculty of Associated Medical Sciences, Chiang Mai University, Chiang Mai 50200, Thailand
}

Received September 22, 2016; Accepted September 22, 2017

DOI: $10.3892 / 01.2017 .7284$

\begin{abstract}
Overexpression of a disintegrin and metalloproteinase 9 (ADAM9) has been shown in various types of cancer. Some studies have reported inconclusive findings regarding chromosomal aberrations in the ADAM9-containing region and ADAM9 expression in oral cancer. Therefore, in this study, ADAM9 protein expression was determined and compared between oral squamous cell carcinoma (OSCC) and normal oral tissues, and between oral cancer cell lines and human oral keratinocytes (HOKs). In total, 34 OSCC and 10 healthy paraffin-embedded tissue sections were probed with an anti-ADAM9 antibody, and the immunohistochemical score was determined by multiplying the percentage of positively stained cells with the intensity score. Four different oral cancer and eight independent HOK cell lines were cultured, and the expression of membrane ADAM9 and active ADAM9 at $84 \mathrm{kDa}$ in these cell lines was assayed by flow cytometry and western blot hybridization, respectively. The results showed that the median immunohistochemical score of ADAM9 expression in OSCC tissues was significantly greater than that in normal tissues $(\mathrm{P}<0.001)$. Furthermore, among OSCC cases, intense staining of ADAM9 expression was detected in well-differentiated and in moderately-differentiated OSCC; ADAM9 expression was also correlated with an increased degree of cell differentiation $(r=0.557 ; \mathrm{P}=0.001)$. Expression of membrane ADAM9 was present in 3/4 cancer cell lines. Expression of active ADAM9 varied among all the tested cell
\end{abstract}

Correspondence to: Dr Suttichai Krisanaprakornkit, Center of Excellence in Oral and Maxillofacial Biology, Department of Oral Biology and Diagnostic Sciences, Faculty of Dentistry, Chiang Mai University, 110 Suthep Road, Chiang Mai 50200, Thailand

E-mail:suttichai.k@cmu.ac.th

Key words: a disintegrin and metalloproteinase 9, cell differentiation, epithelial cells, keratinocytes, oral cancer, squamous cell carcinoma lines, but significantly higher ADAM9 expression was present in certain cancer cell lines than those in HOKs $(\mathrm{P}<0.05)$. In summary, ADAM9 expression is enhanced in OSCC and oral cancer cell lines, suggesting its role in the pathogenesis of oral cancer. Similar to the overexpression of ADAM9 in well-differentiated prostate cancer, high degrees of ADAM9 expression have also been observed in well-differentiated OSCC.

\section{Introduction}

Approximately 300,000 new cases of oral cancer, or $\sim 2.1 \%$ of all cancer cases, were reported worldwide in 2012, with a greater prevalence in males than in females at a ratio of 2:1 (1). Generally, oral squamous cell carcinoma (OSCC) accounts for $\sim 94 \%$ of all oral cancer cases reported (2). OSCC is a devastating disease with 145,000 mortalities $(\sim 1.8 \%$ of the total cancer-associated mortalities) reported in 2012 (1). In Thailand, oral cancer is the sixth most common type of cancer, and there were 3,689 new cases diagnosed in 2012 (1). The conventional treatment for oral cancer includes surgical and radiation therapy, with or without adjuvant chemotherapy $(3,4)$, but the five-year survival rate for patients with OSCC remains low, depending on the Tumor-Node-Metastasis (TNM) staging (5). Consequently, a 'targeted therapy' has been introduced as a possible and adjuvant treatment for patients with cancer (6). An example of a possible target molecule relevant to the present study has been reported in a previous study, which demonstrated that the inhibition of a disintegrin and metalloproteinase 9 (ADAM9) expression in prostate cancer cells in vitro results in enhanced cancer cell death and increased sensitivity to radiotherapy and chemotherapy (7).

ADAM9, otherwise known as metalloproteinase disintegrin cysteine-rich protein 9 or meltrin $\gamma$, was first discovered in 1995 (8). The ADAM9 gene is in the chromosomal region 8p11.23-11.22 (9). ADAM9 is first synthesized as an intracellular pro-form protein with the molecular weight of $110 \mathrm{kDa}$, and is later cleaved to create a mature 84-kDa type I membrane-anchored protein (10). ADAM9 contains several domains, including a propeptide domain, a metalloproteinase 
domain, a disintegrin domain, a cysteine-rich domain, an epidermal growth factor-like domain, a transmembrane domain and a cytoplasmic tail (11). ADAM9 is widely expressed in human tissues (11), especially in the suprabasal layers of the skin, indicating the association between ADAM9 expression and cell differentiation (12). ADAM9 possesses shedding and adhesive properties due to its metalloproteinase and disintegrin domains, respectively (13). Several specific substrates for ADAM9 have been reported, including heparin-binding epidermal growth factor (HB-EGF), $\beta$-amyloid precursor protein, fibronectin, $\beta$-casein, gelatin, tumor necrosis factor (TNF)- $\alpha$, fibroblast growth factor, p75 TNF receptor, and c-kit ligand-1 (14,15). Therefore, ADAM9 can function in both physiological and pathological conditions.

Regarding its involvement in pathologic conditions, ADAM9 overexpression has been identified in a variety of cancer types, including breast cancer (16), renal cancer (17), prostate cancer (18), skin melanoma (19), uterine cervical cancer $(20,21)$, hepatocellular carcinoma (22), non-small cell lung cancer (23), colon cancer (24), gastric cancer (15), esophageal cancer (25) and head and neck squamous cell carcinoma (HNSCC) (26). In the oral cavity, conflicting results of chromosomal aberrations in the $A D A M 9$-containing region have been identified by the array comparative genomic hybridization technique $(9,27)$. Furthermore, no significant difference in ADAM9 mRNA expression has been observed between OSCC and normal tissues, or between oral cancer cell lines and normal oral keratinocytes $(9,28)$. Conversely, a significant difference in ADAM9 mRNA expression has been reported between HNSCC and normal tissues (26). Therefore, these inconclusive data prompted the determination of the ADAM9 protein expression profile in OSCC tissues and oral cancer cell lines. The objectives of the present study were: (1) To compare ADAM9 protein expression between OSCC and normal oral tissue specimens; (2) to determine membrane ADAM9 expression in oral cancer cell lines; (3) to compare ADAM9 protein expression between oral cancer cell lines and normal human oral keratinocytes (HOKs).

\section{Materials and methods}

Antibodies. The goat polyclonal anti-ADAM9 antibody (cat no. sc-23290) and the mouse monoclonal anti- $\beta$-actin antibody (cat no. sc-47778) used in immunohistochemistry and western blot hybridization were from Santa Cruz Biotechnology, Inc. (Dallas, TX, USA). The rabbit anti-ADAM9 antibody (cat no. LS-C100638) used in flow cytometry was from LifeSpan BioSciences, Inc. (Seattle, WA, USA). The phycoerythrin (PE)-conjugated goat $\mathrm{F}\left(\mathrm{ab}^{\prime}\right)_{2}$ anti-rabbit antibody (cat no. P2771MP) was obtained from Invitrogen (Thermo Fisher Scientific, Inc., Waltham, MA, USA).

Immunohistochemistry. A total of 34 formalin-fixed and paraffin-embedded OSCC tissue specimens, including all four clinical stages according to Sobin et al (29), and ten tissue specimens of healthy oral mucosa were retrieved from the tissue archive of the Dental Hospital, Faculty of Dentistry, Chiang Mai University, (Chiang Mai, Thailand). Of 34 OSCC cases, 17 were female and 17 were male, with a mean age of 66.94 years (43-85 years). Other clinicopathological characteristics of the 34 OSCC cases are summarized in Table I. The histological grading of the OSCC specimens was examined by an oral pathologist (Department of Oral Biology and Diagnostic Sciences, Faculty of Dentistry, Chiang Mai University). The research protocol (no. 54/2014) was approved by the Human Experimentation Committee, Faculty of Dentistry, Chiang Mai University. The specimens were sectioned at 5- $\mu \mathrm{m}$ thickness and placed on silanized glass slides (Dako; Agilent Technologies, Inc., Santa Clara, CA, USA). The immunohistochemical protocol used in the present study was modified from that of Iamaroon and Krisanaprakornkit, 2009 (30). Briefly, the tissue sections were deparaffinized in xylene, rehydrated through graded alcohol and distilled water, and incubated with $3 \%$ hydrogen peroxide at room temperature for $10 \mathrm{~min}$. Antigen unmasking was performed in $0.01 \mathrm{M}$ sodium citrate buffer $\mathrm{pH}$ 6.0, prepared from sodium citrate monobasic (cat no. 71497; Sigma-Aldrich; Merck KGaA, Darmstadt, Germany), at $100^{\circ} \mathrm{C}$ for $15 \mathrm{~min}$, and the sections were cooled down at room temperature for $20 \mathrm{~min}$. The sections were blocked with $1.5 \%$ normal blocking serum (cat no. sc-2023; ImmunoCruz ABC Staining Systems; Santa Cruz Biotechnology, Inc.) in Tris-buffered saline (TBS) for $20 \mathrm{~min}$ at room temperature, and then incubated overnight in $100 \mu \mathrm{l}$ goat polyclonal anti-ADAM9 antibody diluted in TBS $(1: 100)$ at $4^{\circ} \mathrm{C}$. For the negative control, the sections were incubated in normal blocking serum without the addition of primary antibody. The sections were subsequently reacted with the secondary anti-goat antibody (cat no. sc-2023; ImmunoCruz ABC Staining Systems; Santa Cruz Biotechnology, Inc.) diluted in PBS (1:100), and then with avidin-biotinylated horseradish peroxidase (ImmunoCruz ABC Staining Systems; Santa Cruz Biotechnology, Inc.) for $20 \mathrm{~min}$ at room temperature. The sections were washed and developed in 3,3'-diaminobenzidine (Vector Laboratories, Inc., Burlingame, CA, USA). The reaction was stopped in water when brown staining was observed without background staining, monitored using light microscopy (CHK-H model; Olympus Corporation, Tokyo, Japan). Subsequently the sections were counterstained with hematoxylin and mounted. Digitized images of the tissue sections were captured by a charge-coupled device (CCD) camera (DP71; Olympus Corporation), attached to a bright-field microscope (BX41; Olympus Corporation) and a desktop computer system.

Determination of immunohistochemical (IHC) score. The sections were scored by two independent observers, with kappa values equal to 0.908 and 0.848 for the intra-examiner and the inter-examiner calibrations, respectively. The observers were blinded to the clinicopathological data for each specimen. ImageJ software (version 1.48; National Institutes of Health, Bethesda, MA, USA) was used to score the number of positively stained cells, regardless of their staining intensity, under x400 magnification. Prior to scoring, each section was observed under x100 magnification to determine the orientation of the epithelium and connective tissue. In normal tissues, scoring was performed only in the epithelial layer; in oral cancer tissues, scoring was performed in the epithelial cell nest in the connective tissue layer. Each section was first screened and then selected for three representative fields of vision. The staining intensities were scored as follows: $0=$ none, $1=$ weak, 
Table I. Clinicopathologic characteristics of OSCC cases.

\begin{tabular}{lc}
\hline Variables & Number of cases \\
\hline Clinical diagnostic staging & \\
I & 6 \\
II & 6 \\
III & 5 \\
IV & 17 \\
Histological grading & \\
Well-differentiated & 14 \\
Moderately-differentiated & 12 \\
Poorly-differentiated & 8 \\
Location & \\
Buccal mucosa & 10 \\
Lateral tongue & 5 \\
Gingiva/alveolar mucosa & 13 \\
Labial mucosa & 1 \\
Retromolar pad & 1 \\
Lip vermillion & 2 \\
Mandible & 2 \\
\hline
\end{tabular}

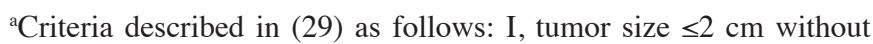
regional lymph node spreading; II, tumor size $>2-4 \mathrm{~cm}$ without regional lymph node spreading; III, tumor size $>4 \mathrm{~cm}$ or of any size with spread to a single ipsilateral lymph node $(\leq 3 \mathrm{~cm})$; IV, tumor of any size with spread to $\geq 1$ ipsilateral lymph node $(>3 \mathrm{~cm})$ or with spread to $\geq 1$ contralateral lymph node, or tumor invasion to adjacent organs or spreading to other parts of the body regardless of lymph node involvement.

$2=$ moderate, and $3=$ intense staining (31). The percentage of positive cells was calculated by dividing against the total number of cells in each field of view. Then, the average percentage of positive cells in each section was determined. Finally, the average percentage of positive cells was multiplied by the staining intensity score, and the IHC score (0-3) was obtained for each section (25).

Cell culture. Four oral cancer cell lines, including HN5, HN6, HN15 and HN008, have been previously studied for the overexpression and activation of Akt2 (30). Primary HOKs were isolated from non-inflamed oral tissues overlying the impacted third molars of eight healthy and non-smoking donors $(n=8)$, with their informed consent, as previously described (30). As a positive control cell line for expression of both pro-form and active form of ADAM9 (32), the hepatoblastoma cell line, HepG2, was obtained from Professor Prachya Kongtawelert, Thailand Excellence Center for Tissue Engineering and Stem Cells, Department of Biochemistry, Faculty of Medicine, Chiang Mai University. HOKs were cultured in serum-free keratinocyte growth medium (Lonza, Walkersville, MA, USA), while the four oral cancer cell lines and HepG2 cells were cultured in Dulbecco's modified Eagle's medium (Invitrogen; Thermo Fisher Scientific, Inc.), supplemented with $10 \%$ fetal bovine serum and $1 \%$ penicillin/streptomycin (Invitrogen; Thermo Fisher Scientific, Inc.). All cells were maintained at $37^{\circ} \mathrm{C}$ in a humidified chamber with $5 \% \mathrm{CO}_{2}$. Culture medium was changed every two days, until the cells reached $80 \%$ confluency, prior to flow cytometry and western blot hybridization.

Flow cytometry. To determine the expression of membrane ADAM9 in four cancer cell lines and HepG 2 cells, flow cytometry was performed. In brief, the tested cells ( $2 \times 10^{6}$ cells) were washed with PBS twice, removed from 100-mm culture dishes by scraping in $0.5 \mathrm{mM}$ EDTA $\mathrm{pH} 7.3$, centrifuged at $400 \mathrm{x} \mathrm{g}$ at room temperature for $5 \mathrm{~min}$, and again washed twice with PBS pH 7.2. To block nonspecific Fc-receptor-mediated antibody binding, cells were pre-incubated with $10 \%$ AB serum from a healthy donor with blood group $\mathrm{AB}$ (from whom written informed consent was obtained), at $4^{\circ} \mathrm{C}$ for $1 \mathrm{~h}$. The blocked cells were then incubated for $30 \mathrm{~min}$ at $4^{\circ} \mathrm{C}$ with the anti-ADAM9 antibody (1:20) or the rabbit immunoglobulins (1:20; final concentration, $20 \mu \mathrm{g} / \mathrm{ml}$ ); normal rabbit serum, which was purified using a protein $\mathrm{G}$ column, was used as the control. The cells that were not incubated with primary antibodies or purified rabbit immunoglobulins served as a negative conjugate control. The cells were washed twice with FACS buffer, PBS containing $1 \%$ bovine serum albumin (code A7906; Sigma-Aldrich) and 0.02\% sodium azide (code 0639; Amresco LLC, Solon, OH, USA), and then incubated with the PE-conjugated goat anti-rabbit immunoglobulin antibody (dilution, 1:1,000) at $4^{\circ} \mathrm{C}$ for $30 \mathrm{~min}$. The fluorescent signals were analyzed by a FACSCalibur flow cytometer (BD Biosciences, San Jose, CA, USA).

Western blot hybridization. Whole cell lysates from all cell lines were extracted using radio-immunoprecipitation assay (RIPA) buffer (33). The total protein was quantified using a BCA Protein Assay kit (Pierce; Thermo Fisher Scientific, Inc.) according to the manufacturer's protocol. The whole cell lysates $(20 \mu \mathrm{g})$ were denatured by heating at $100^{\circ} \mathrm{C}$ for $5 \mathrm{~min}$, then resolved via 7.5\% SDS-PAGE prior to transfer to a nitrocellulose membrane (Bio-Rad Laboratories, Inc., Hercules, CA, USA). Unoccupied binding sites on the membranes were blocked with 5\% non-fat dry milk (Santa Cruz Biotechnology, Inc.) at room temperature for $1 \mathrm{~h}$. Then, the membranes were incubated overnight with the anti-ADAM9 antibody (dilution, $1: 500$ ) or the mouse monoclonal anti- $\beta$-actin antibody (dilution, $1: 1,000)$ at $4^{\circ} \mathrm{C}$. The membranes were subsequently incubated for $1 \mathrm{~h}$ at room temperature with either HRP-conjugated mouse anti-goat immunoglobulins (cat no. 31400; Thermo Fisher Scientific, Inc.) or HRP-conjugated rabbit anti-mouse immunoglobulins (cat no. P0260; Dako; Agilent Technologies) at 1:2,000 dilutions. The LumiGLO Reserve ${ }^{\circledR}$ chemiluminescent reagent (KPL, Gaithersburg, MA, USA) was used as a substrate, and the signals were captured using a CCD camera attached to the ChemiDoc ${ }^{\mathrm{TM}}$ XRS gel documentation system (Bio-Rad Laboratories, Inc.). To quantify the expression of active ADAM9 in each cell line, the intensities of the bands at $84 \mathrm{kDa}$ were measured using the Scion Image program (Scion Corporation, Frederick, MA, USA), and the intensity of ADAM9 expression was normalized to that of $\beta$-actin expression in each sample. Then, the ratios of ADAM9 to $\beta$-actin expression were adjusted for the percentages of ADAM9 expression by comparing the ratio of ADAM9 to $\beta$-actin 
Table II. A semi-quantitative analysis of the intensity score for ADAM9 expression in OSCC, according to different levels of histological grading.

\begin{tabular}{lccc}
\hline Intensity score $^{\mathrm{a}}$ & Well-differentiated N $(\%)$ & Moderately-differentiated N $(\%)$ & Poorly-differentiated N $(\%)$ \\
\hline 0 & $0(0)$ & $0(0)$ & $0(0)$ \\
1 & $2(14.3)$ & $1(8.3)$ & $3(37.5)$ \\
2 & $3(21.4)$ & $8(66.7)$ & $5(62.5)$ \\
3 & $9(64.3)$ & $3(25)$ & $0(0)$ \\
Total & $14(100)$ & $12(100)$ & $8(100)$ \\
\hline
\end{tabular}

${ }^{a} 0$, no staining; 1, weak (light brown staining, visible only with high magnification); 2, moderate (between 1 and 3); 3 , intense (dark brown staining, visible with low magnification) (31). ADAM9, a disintegrin and metalloproteinase 9; OSCC, oral squamous cell carcinoma.

expression in each cell line with the highest ratio of ADAM9 to $\beta$-actin expression observed in HN008 cells, set to $100 \%$.

Statistical analysis. The Mann-Whitney U test was used to compare the IHC scores, representing ADAM9 expression, between the OSCC and normal oral tissues. The Spearman's correlation coefficient was used to determine the correlations between the IHC scores and the histological grading or clinical staging. The independent sample t-test was used to compare the percentages of ADAM9 expression between oral cancer cell lines and normal HOKs. The data from the Mann-Whitney $\mathrm{U}$ test are presented as medians and interquartile ranges; the data from the independent sample t-test are presented as means and standard deviations. All statistical analyses were carried out with SPSS software version 17 (SPSS, Inc., Chicago, IL, USA). $\mathrm{P}<0.05$ was considered to indicate a statistically significant difference.

\section{Results}

Overexpression of ADAM9 in OSCC. Using immunohistochemistry, intense ADAM9 staining was observed in both the cytoplasm and the membrane of the majority of cancer cell nests, which were localized in the connective tissue layer of OSCC specimens (arrowheads in Fig. 1A); comparatively, weak and diffuse ADAM9 staining was observed in the cytoplasm of normal epithelial cells, particularly in the suprabasal cell layers (Fig. 1A). The intensity of ADAM9 staining in the overlying epithelium of the OSCC tissues was also weak (bracket in Fig. 1A). No staining in the OSCC sections was observed in the absence of the anti-ADAM9 antibody (data not presented). The median IHC score in the OSCC group was significantly higher than that in the normal group $(\mathrm{P}<0.001$; Fig. 1B).

Positive correlation between ADAM9 expression and cell differentiation in OSCC. All 34 OSCC cases were grouped into four clinical diagnostic stages (Table I) according to tumor size and nodal status (29), based on the histopathological reports. Using the Spearman's correlation test, the IHC scores, representing ADAM9 expression, were not determined to correlate with these four clinical diagnostic stages $(r=-0.082, \mathrm{P}=0.661)$, indicating that ADAM9 protein expression in OSCC has no significant association with OSCC severity and aggressiveness.
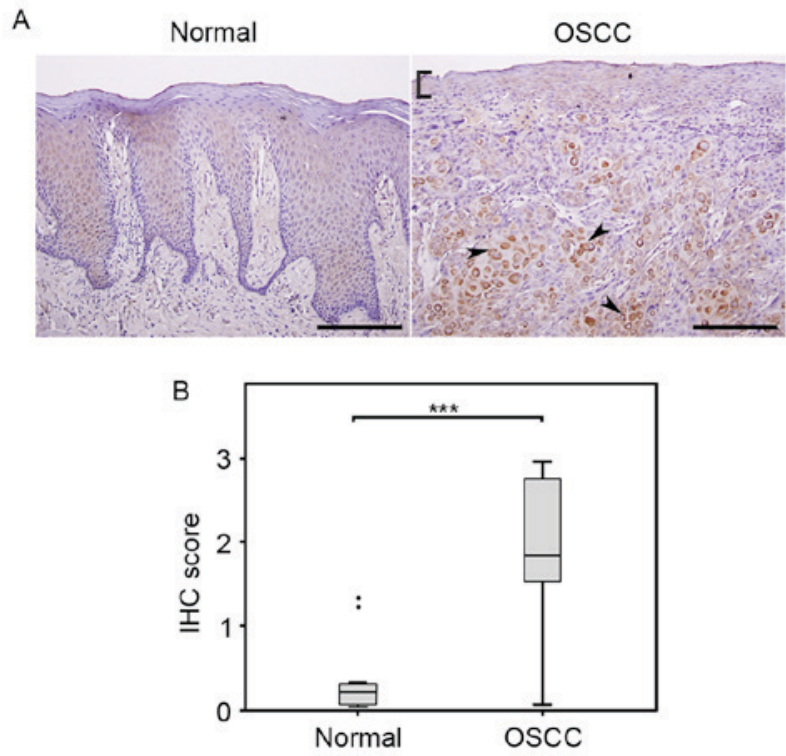

Figure 1. ADAM9 overexpression in OSCC. (A) A representative image of ADAM9 expression in normal and OSCC tissues. Weak ADAM9 staining is present in the suprabasal layers of normal epithelium and in the overlying epithelium of OSCC (bracket); intense cytoplasmic staining of ADAM9 is in tumor cell nests within the connective tissue layer of OSCC (arrowheads). Bar=200 micron. (B) A box plot diagram demonstrating significantly higher IHC scores (0-3) for ADAM9 expression in the OSCC group than in the normal group. ${ }^{* * *} \mathrm{P}<0.001$. Two small black circles represent outliers in the normal group. ADAM9, a disintegrin and metalloproteinase 9; OSCC, oral squamous cell carcinoma; IHC, immunohistochemical.

However, when all OSCC cases were stratified according to their histological grade, including 14 well-differentiated, 12 moderately-differentiated and 8 poorly-differentiated cases (Table I), it was revealed that the intensity of ADAM9 staining was greatest in the well-differentiated OSCC tissues, followed by in the moderately and the poorly-differentiated OSCC tissues, respectively (Fig. 2A). A semi-quantitative analysis of the staining intensity in all OSCC specimens is summarized in Table II. In general, the intense score $(=3)$ was applicable in $>60 \%$ of the well-differentiated OSCC cases, whereas it was assigned in only 25 and $0 \%$ of the moderately and the poorly-differentiated OSCC cases, respectively (Table II). By contrast, $>60 \%$ of the moderately and the poorly-differentiated OSCC cases were scored as moderate staining (=2). Similar to the staining intensity scores, the average percentage of 
A
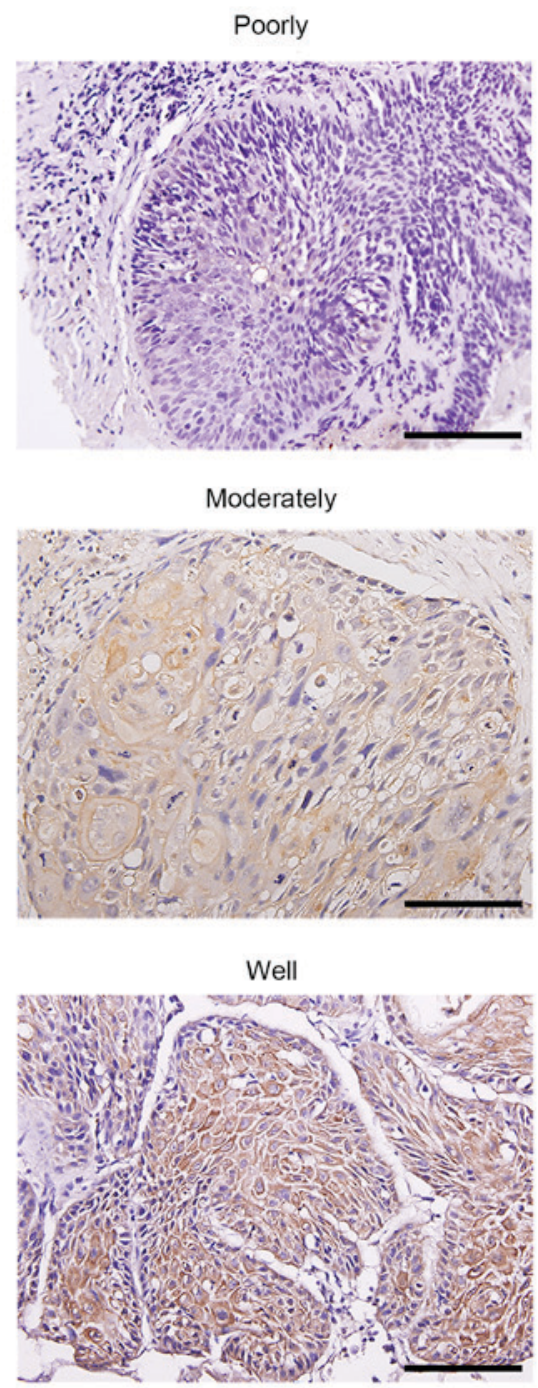

B

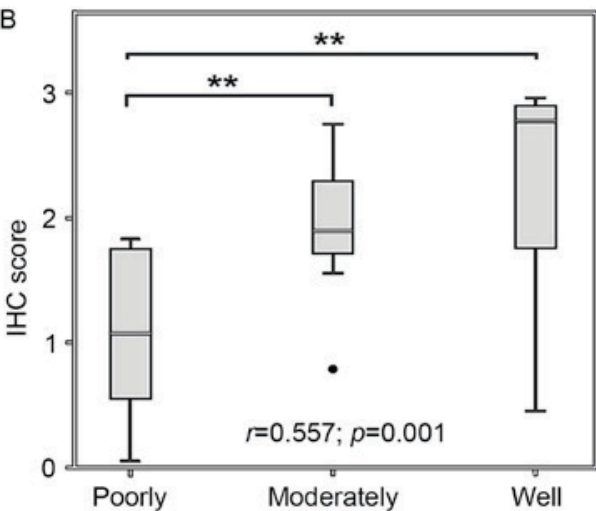

Figure 2. A positive correlation between ADAM9 expression and cell differentiation in OSCC. (A) A representative image of ADAM9 expression in each histological grading. Note the strongest intensity of ADAM9 staining in the well-differentiated OSCC, followed by the moderately-differentiated and the poorly-differentiated OSCC. Bar $=100$ micron. (B) A box plot diagram showing the positive correlation between increased IHC scores (0-3) for ADAM9 expression and enhanced levels of cell differentiation. ${ }^{* *} \mathrm{P}<0.01$. A small black circle represents an outlier in the moderately-differentiated OSCC. ADAM9, a disintegrin and metalloproteinase 9; OSCC, oral squamous cell carcinoma; IHC, immunohistochemical.

positively-stained cells, irrespective of staining intensity, was greater in the well and in the moderately-differentiated OSCC tissues than in the poorly-differentiated OSCC (data not presented). Using the Spearman's correlation test, the IHC scores were positively correlated with an increased degree of cell differentiation $(r=0.557, \mathrm{P}=0.001$; Fig. $2 \mathrm{~B})$. Therefore, the median IHC scores for the moderately and well-differentiated OSCC tissues were significantly higher than that of the poorly-differentiated OSCC $(\mathrm{P}<0.01$; Fig. $2 \mathrm{~B})$. However, there was no significant difference in the median IHC score between the well and moderately-differentiated OSCC (Fig. 2B), suggesting that ADAM9 expression may not be used as a diagnostic marker to distinguish well-differentiated OSCC from moderately-differentiated OSCC.

Significantly increased expression of active ADAM9 in oral cancer cell lines. We first determined the expression of membrane ADAM9 in four different oral cancer cell lines, HN5, HN6, HN15 and HN008, by flow cytometry. It was found that membrane ADAM9 was expressed in 3/4 tested oral cancer cell lines, HN6, HN15 and HN008, whereas membrane ADAM9 was not expressed in HN5 cells (Fig. 3). As expected, membrane ADAM9 was expressed in HepG2 cells. No expression signals were detected in any of the tested cell lines, either those incubated with the purified rabbit immunoglobulins control or without the anti-ADAM9 antibody (Fig. 3). Varying degrees of ADAM9 expression for the pro-form, previously reported as 110 or $120 \mathrm{kDa}(10,34)$, and for the active form (84 kDa) were detected in the whole cell lysates of HN5, HN6, HN15, HN008 and 8 independent HOK cell lines (Fig. 4A). In general, the expression of active ADAM9 in HN6, HN15 and HN008 cells was greater than that in HOKs, whereas the expression of the active form of ADAM9 in HN5 cells was less than that in the HOKs (Fig. 4A), which is consistent with the absence of a fluorescent signal for membrane ADAM9 in HN5 cells (Fig. 3). Expression of $\beta$-actin was equivalent among the various samples (Fig. 4A). Using densitometry, the average percentage expression of the active form of ADAM9 in HN6 and HN008 cells was significantly higher than that in the 8 independent HOKs $(\mathrm{P}<0.05$ and $\mathrm{P}<0.001$, respectively); however, there was no significant increase in the expression of the active form of ADAM9 in HN15 cells (Fig. 4B). By contrast, the mean percentage expression of the active form of ADAM9 in HN5 cells was significantly lower than that in HOKs ( $\mathrm{P}<0.001$; Fig. 4B).

\section{Discussion}

In this study, a significant increase in ADAM9 protein expression in OSCC tissues, compared with in normal oral tissues, was demonstrated by immunohistochemistry, a finding that agrees with the results of other prior studies, which demonstrated ADAM9 protein overexpression in various types of cancer $(9,15,17,18,20-23,25,26)$. Though particularly in squamous cell carcinoma, ADAM9 protein overexpression is also present in cancer of the cervix, the esophagus, the pharynx, the larynx, and the skin around the head and neck region $(9,21,25,26)$. However, few previous studies have demonstrated no significant difference in ADAM9 mRNA expression between OSCC and normal tissues, or between oral cancer cell lines and normal oral keratinocytes $(9,28)$, a finding that differs from our results on ADAM9 protein overexpression. This discrepancy may suggest a possible post-transcriptional or epigenetic modification of 

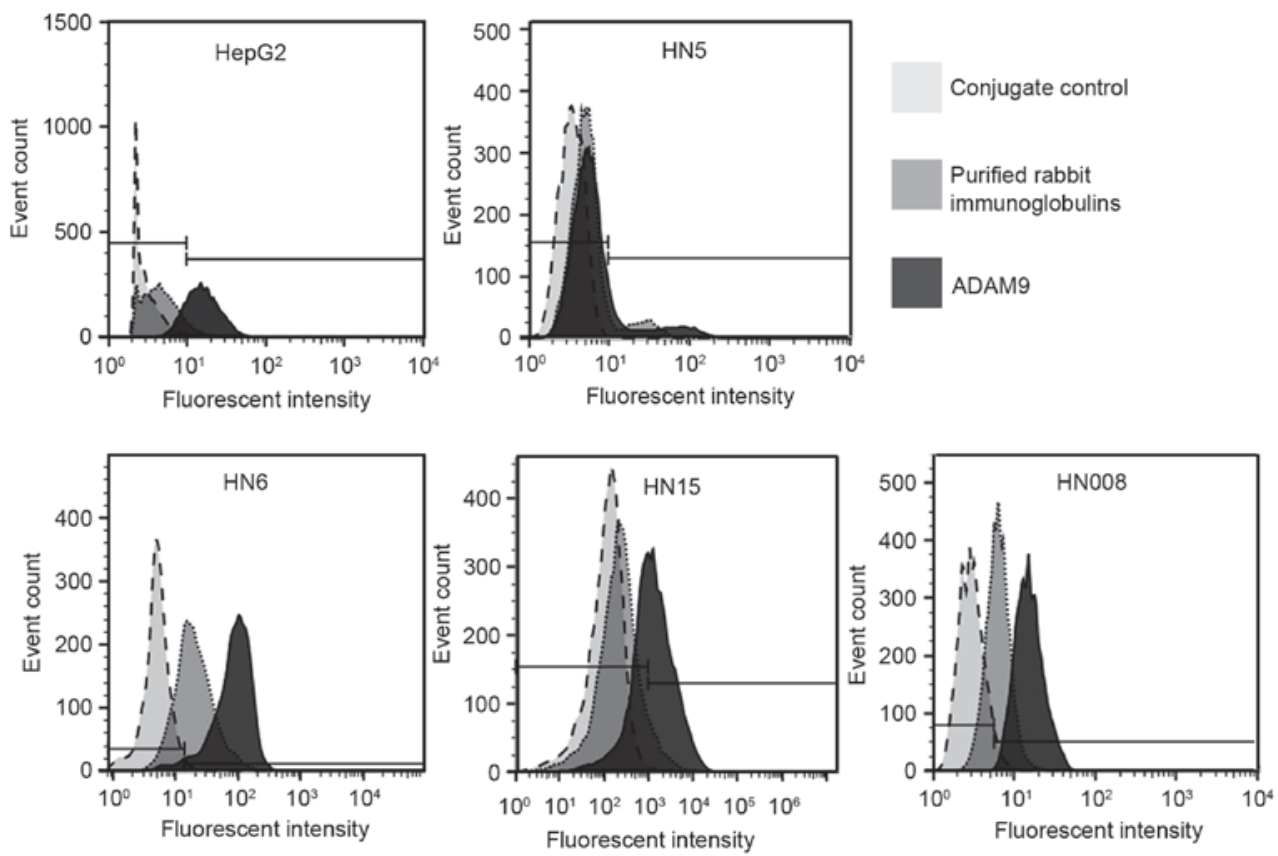

Figure 3. Expression of membrane ADAM9 in oral cancer cell lines. A representative histogram for expression of membrane ADAM9 in the four oral cancer cell lines, HN5, HN6, HN15 and HN008, from three separate experiments is presented. Note the expression of membrane ADAM9 (black area) in HN6, HN15 and HN008 cells, whereas membrane ADAM9 expression was not detected in HN5 cells. Membrane ADAM9 was expressed in HepG2 cells, which were used as the positive control, while there was no membrane ADAM9 expression in all four oral cancer cell lines and HepG2 cells stained with only the PE-conjugated goat anti-rabbit immunoglobulin antibody as the conjugate control (light gray area) or with purified rabbit immunoglobulins (dark gray area), used as the two negative controls. ADAM9, a disintegrin and metalloproteinase 9 .

A

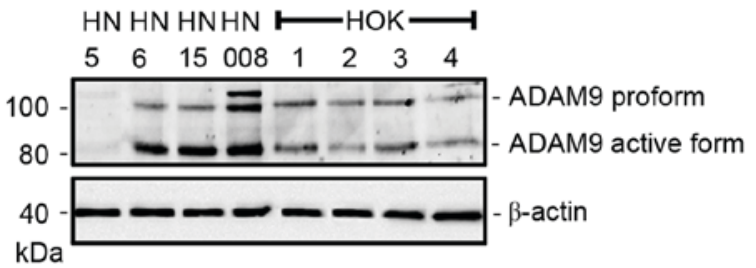

B

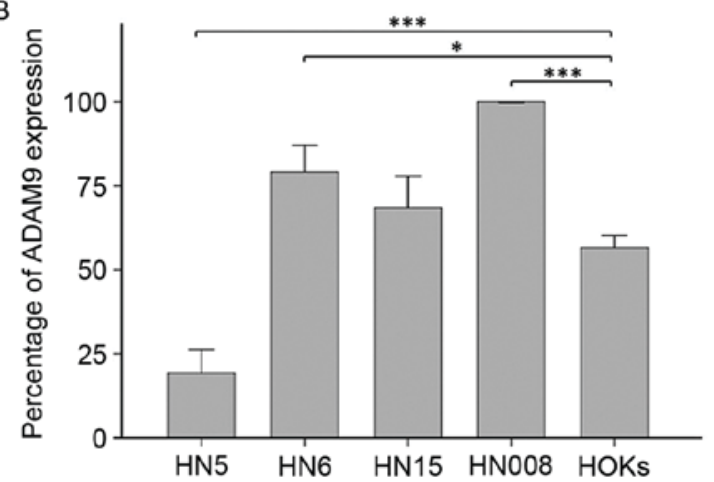

Figure 4. A significant increase in the expression of active ADAM9 in certain oral cancer cell lines. (A) A representative blot demonstrating varying expression of the ADAM9 pro-form (110 and $120 \mathrm{kDa})$ and of its active form (84 kDa) in the four oral cancer cell lines, HN5, HN6, HN15 and HN008, and in four normal human oral keratinocytes (HOK 1-4). Expression of $\beta$-actin was equal among all the samples. (B) A bar graph demonstrating a significant increase in the average percentage of active ADAM9 expression in HN6 and HN008 cells, and a significant decrease in the average percentage in HN5 cells, determined from five separate experiments $(n=5)$ and compared with the average percentage of ADAM9 expression in eight independent HOK cell lines $(\mathrm{n}=8)$. The percentage of ADAM9 expression in each oral cancer and HOK cell line was determined by comparison with the percentage in HN008 cells, set to $100 \%$. Error bars=standard deviations; ${ }^{*} \mathrm{P}<0.05 ;{ }^{* * * *} \mathrm{P}<0.001$. ADAM9, a disintegrin and metalloproteinase 9; HOK, human oral keratinocytes.
ADAM9 expression, as demonstrated in esophageal squamous cell carcinoma (25).

A significant and positive correlation was demonstrated between ADAM9 expression and an increased degree of oral cancer cell differentiation. This finding is similar to the results shown in mouse prostate and breast cancer, wherein higher ADAM9 mRNA expression is present in well-differentiated lesions, compared with in poorly-differentiated lesions (35). Moreover, the significantly greater ADAM9 protein expression in well and moderately-differentiated OSCC, compared with in poorly-differentiated OSCC, may suggest a possible role for ADAM9 during the transition from well-differentiated to poorly-differentiated carcinomas, as proposed by Peduto et al (35). Poorly-differentiated OSCC is generally associated with cancer aggressiveness due to its high recurrence rate and poor disease-free survival rate, compared with well and moderately-differentiated OSCC (36). As a result, it is speculated that decreased ADAM9 expression in OSCC may regulate a change from low-grade to high-grade cancer that then results in increased cancer aggressiveness and severity.

The significantly increased ADAM9 protein expression in well and in moderately-differentiated OSCC also corresponds with ADAM9 expression in the suprabasal layers of normal epidermis and in differentiated skin keratinocyte cell lines (12), indicating the association between ADAM9 expression and epithelial cell differentiation in both OSCC and normal tissues. However, the present study determined that ADAM9 expression in OSCC was not correlated with tumor size or nodal status, suggesting that the degree of ADAM9 expression is not involved with the clinical features or anatomical extent of oral cancer. A lack of this involvement is consistent with higher ADAM9 expression in well-differentiated OSCC compared 
with in poorly-differentiated OSCC (Fig. 2), as well as with no association observed between ADAM9 expression and the TNM staging of prostate and lung cancer $(18,23)$. Nonetheless, the correlation results were based on the histopathological reports of 34 OSCC cases. A notable limitation of the present study was the lack of treatment and follow-up data for the 34 patients with OSCC; it would, therefore, be useful to further determine whether distinct degrees of ADAM9 expression are associated with OSCC aggressiveness, particularly the recurrence and the five-year survival rates.

Consistent with ADAM9 protein expression on the surface of cancer cells in OSCC tissues, expression of membrane ADAM9 was detected in 3/4 tested oral cancer cell lines using flow cytometry (Fig. 3). Furthermore, using western blot hybridization, the expression of membrane ADAM9 (active form) at $84 \mathrm{kDa}$ was significantly enhanced in $2 / 3$ oral cancer cell lines (Fig. 4B), which were positive for membrane ADAM9 expression. These results are similar to those of Uehara et al (28), who demonstrated increased ADAM9 mRNA expression in certain oral cancer cell lines, and also add up their results (28) by demonstrating the presence of membrane ADAM9 in oral cancer cell lines and a significant increase in membrane ADAM9 expression, suggesting that ADAM9 can possibly function as a shedding enzyme for HB-EGF $(14,25)$. In addition, varying degrees of active ADAM9 expression among oral cancer and HOK cell lines are similar to the different degrees of ADAM9 mRNA expression in other oral cancer and normal epithelial cell lines (28).

ADAM9 overexpression has been demonstrated to serve an essential role in the pathogenesis of numerous cancer types, particularly through enhanced cancer cell survival due to an anti-apoptotic activity mediated by the epidermal growth factor receptor (EGFR)/Akt pathway in squamous cell carcinoma of the skin and the esophagus $(19,25)$. The active ADAM9 overexpression in oral cancer cell lines is concordant with the results of our previous study (30), which recorded the overexpression and activation of Akt 2 in the same oral cancer cell lines. Thus, the possible function of active ADAM9 as a sheddase that activates EGFR and Akt via phosphorylation in these oral cancer cell lines warrants further investigation. It is also necessary to examine the behavior and aggressiveness of the HN6 and HN008 cells, in which ADAM9 expression was significantly enhanced compared with HN5 cells that minimally expressed ADAM9. Further investigations into the effect of ADAM9 inhibition in both normal oral cells and highly expressing ADAM9 oral cancer cell lines are also required.

As ADAM9 does not appear to be essential to development, fertility or adult homeostasis, inhibition of ADAM9 expression in tumors may only affect cancer cells, and not normal cells, resulting in fewer complications than conventional therapies, including surgical, chemo and radiation therapies (37). Recently, studies have proposed the usage of ADAM9 as a prognostic or a therapeutic marker in various types of cancer. One example is a study conducted using high and low ADAM9-expressing gastric cancer cell lines (15). It was observed that monoclonal antibodies, or small interfering RNA, specifically targeted against ADAM9 could reduce cell proliferation and invasion in high ADAM9-expressing cell lines but not in low ADAM9-expressing cell lines (15). Therefore, it is reasonable to assume that silencing ADAM9 expression could prove useful in decreasing aggressive behavior in high ADAM9-expressing oral cancer cell lines, which warrants further investigation.

In summary, the present study is the first, to the best of our knowledge, to demonstrate that ADAM9 protein is overexpressed in OSCC tissues and in oral cancer cell lines. Its expression is positively correlated with cancer cell differentiation, consistent with other cancer types and with increased ADAM9 expression in the suprabasal layers of the skin. Nevertheless, ADAM9 overexpression in OSCC is not correlated with tumor size or nodal status, which corresponds with low levels of ADAM9 expression in poorly-differentiated OSCC tissues. Certain oral cancer cell lines that highly express active ADAM9 on their cell surface also strongly express Akt2 and phosphorylated-Akt (30), suggesting a possible connection between active ADAM9 and the activation of EGFR and Akt2 via phosphorylation in these oral cancer cell lines. Additional studies are still required to explore the functional role of ADAM9 in the pathogenesis and aggressiveness of OSCC.

\section{Acknowledgements}

The present study was supported by the Intramural Endowment Fund, Faculty of Dentistry, Chiang Mai University to Dr Pattaramon Tanasubsinn; the PhD scholarship (PHD/013/2557) from Chiang Mai University to Dr Win Pa Pa Aung; the Thailand Research Fund (no. BRG6080001) to Dr Suttichai Krisanaprakornkit; and the TRF Senior Research Scholar (no. RTA5980007) to Dr Watchara Kasinrerk. The authors thank Dr M. Kevin O Carroll, Professor Emeritus of the University of Mississippi School of Dentistry, USA, and Faculty Consultant at Chiang Mai University Faculty of Dentistry, Thailand, for his critical reading of this manuscript.

\section{References}

1. Ferlay J, Soerjomataram I, Dikshit R, Eser S, Mathers C, Rebelo M, Parkin DM, Forman D and Bray F: Cancer incidence and mortality worldwide: Sources, methods and major patterns in GLOBOCAN 2012. Int J Cancer 136: E359-E386, 2015.

2. Neville B, Damm DD, Allen C and Bouquot J: Oral and Maxillofacial Pathology 4th edition. Elsevier Health Sciences, Canada, pp409-421, 2009.

3. Deng H, Sambrook PJ and Logan RM: The treatment of oral cancer: An overview for dental professionals. Aust Dent J 56: 244-252, 2011

4. Rosebush MS, Rao SK, Samant S, Gu W, Handorf CR, Pfeffer LM and Nosrat CA: Oral cancer: Enduring characteristics and emerging trends. J Mich Dent Assoc 94: 64-68, 2012.

5. Warnakulasuriya S: Global epidemiology of oral and oropharyngeal cancer. Oral Oncol 45: 309-316, 2009.

6. Howard JD, Lu B and Chung CH: Therapeutic targets in head and neck squamous cell carcinoma: Identification, evaluation, and clinical translation. Oral Oncol 48: 10-17, 2012.

7. Josson S, Anderson CS, Sung SY, Johnstone PA, Kubo H, Hsieh CL, Arnold R, Gururajan M, Yates C and Chung LW: Inhibition of ADAM9 expression induces epithelial phenotypic alterations and sensitizes human prostate cancer cells to radiation and chemotherapy. Prostate 71: 232-240, 2011.

8. Barrett A, Woessner J and Rawlings N (eds): Handbook of Proteolytic Enzymes 2nd edition. Elsevier Inc, Europe, pp715, 2013.

9. Vincent-Chong VK, Anwar A, Karen-Ng LP, Cheong SC, Yang YH, Pradeep PJ, Rahman ZA, Ismail SM, Zaini ZM, Prepageran N, et al: Genome wide analysis of chromosomal alterations in oral squamous cell carcinomas revealed over expression of MGAM and ADAM9. PLoS One 8: e54705, 2013. 
10. Roychaudhuri R, Hergrueter AH, Polverino $F$, Laucho-Contreras ME, Gupta K, Borregaard N and Owen CA: ADAM9 is a novel product of polymorphonuclear neutrophils: Regulation of expression and contributions to extracellular matrix protein degradation during acute lung injury. J Immunol 193: 2469-2482, 2014.

11. Hooper NM and Lendeckel U (eds): The ADAM family of protease. Springer, The Netherlands, pp75-81, 2005.

12. Zigrino P, Steiger J, Fox JW, Löffek S, Schild A, Nischt R and Mauch C: Role of ADAM-9 disintegrin-cysteine-rich domains in human keratinocyte migration. J Biol Chem 282: 30785-30793, 2007.

13. Sung SY: ADAM9 (ADAM metallopeptidase domain 9 (meltrin gamma)). Atlas Genet Cytogenet Oncol Haematol 14: 270-274, 2010.

14. Klein T and Bischoff R: Active metalloproteases of the A Disintegrin and Metalloprotease (ADAM) family: Biological function and structure. J Proteome Res 10: 17-33, 2011.

15. Kim JM, Jeung HC, Rha SY, Yu EJ, Kim TS, Shin YK, Zhang X, Park KH, Park SW, Chung HC and Powis G: The effect of disintegrin-metalloproteinase ADAM9 in gastric cancer progression. Mol Cancer Ther 13: 3074-3085, 2014.

16. Fry JL and Toker A: Secreted and membrane-bound isoforms of protease ADAM9 have opposing effects on breast cancer cell migration. Cancer Res 70: 8187-8198, 2010.

17. Fritzsche FR, Wassermann K, Jung M, Tölle A, Kristiansen I, Lein M, Johannsen M, Dietel M, Jung K and Kristiansen G: ADAM9 is highly expressed in renal cell cancer and is associated with tumour progression. BMC Cancer 8: 179, 2008.

18. Fritzsche FR, Jung M, Tölle A, Wild P, Hartmann A, Wassermann K, Rabien A, Lein M, Dietel M, Pilarsky C, et al: ADAM9 expression is a significant and independent prognostic marker of PSA relapse in prostate cancer. Eur Urol 54: 1097-1106, 2008.

19. Singh B, Schneider M, Knyazev P and Ullrich A: UV-induced EGFR signal transactivation is dependent on proligand shedding by activated metalloproteases in skin cancer cell lines. Int J Cancer 124: 531-539, 2009

20. Zubel A, Flechtenmacher C, Edler L and Alonso A: Expression of ADAM9 in CIN3 lesions and squamous cell carcinomas of the cervix. Gynecol Oncol 114: 332-336, 2009.

21. Shaker M, Yokoyama Y, Mori S, Tsujimoto M, Kawaguchi N, Kiyono T, Nakano T and Matsuura N: Aberrant expression of disintegrin-metalloprotease proteins in the formation and progression of uterine cervical cancer. Pathobiology 78: 149-161, 2011.

22. Tao K, Qian N, Tang Y, Ti Z, Song W, Cao D and Dou K: Increased expression of a disintegrin and metalloprotease-9 in hepatocellular carcinoma: Implications for tumor progression and prognosis. Jpn J Clin Oncol 40: 645-651, 2010.

23. Zhang J, Qi J, Chen N, Fu W, Zhou B and He A: High expression of a disintegrin and metalloproteinase-9 predicts a shortened survival time in completely resected stage I non-small cell lung cancer. Oncol Lett 5: 1461-1466, 2013.

24. Li J, Ji Z, Qiao C, Qi Y and Shi W: Overexpression of ADAM9 promotes colon cancer cells invasion. J Invest Surg 26: 127-133, 2013.
25. Liu R, Gu J, Jiang P, Zheng Y, Liu X, Jiang X, Huang E, Xiong S, Xu F, Liu G, et al: DNMT1-microRNA126 epigenetic circuit contributes to esophageal squamous cell carcinoma growth via ADAM9-EGFR-AKT signaling. Clin Cancer Res 21: 854-863, 2015.

26. Stokes A, Joutsa J, Ala-Aho R, Pitchers M, Pennington CJ, Martin C, Premachandra DJ, Okada Y, Peltonen J, Grénman R, et al: Expression profiles and clinical correlations of degradome components in the tumor microenvironment of head and neck squamous cell carcinoma. Clin Cancer Res 16: 2022-2035, 2010

27. Ambatipudi S, Gerstung M, Gowda R, Pai P, Borges AM, Schäffer AA, Beerenwinkel N and Mahimkar MB: Genomic profiling of advanced-stage oral cancers reveals chromosome $11 \mathrm{q}$ alterations as markers of poor clinical outcome. PLoS One 6: e17250, 2011.

28. Uehara E, Shiiba M, Shinozuka K, Saito K, Kouzu Y, Koike H, Kasamatsu A, Sakamoto Y, Ogawara K, Uzawa K and Tanzawa H: Upregulated expression of ADAM12 is associated with progression of oral squamous cell carcinoma. Int J Oncol 40: 1414-1422, 2012.

29. Sobin LH, Gospodarowicz MK and Wittekind C: TNM classification of malignant tumours. Blackwell Publishing Ltd, United Kingdom, pp5-29, 2009.

30. Iamaroon A and Krisanaprakornkit S: Overexpression and activation of Akt 2 protein in oral squamous cell carcinoma. Oral Oncol 45: e175-e179, 2009.

31. Pirker R, Pereira JR, von Pawel J, Krzakowski M, Ramlau R, Park K, de Marinis F, Eberhardt WE, Paz-Ares L, Störkel S, et al: EGFR expression as a predictor of survival for first-line chemotherapy plus cetuximab in patients with advanced non-small-cell lung cancer: Analysis of data from the phase 3 FLEX study. Lancet Oncol 13: 33-42, 2012.

32. Kohga K, Takehara T, Tatsumi T, Ishida H, Miyagi T, Hosui A and Hayashi N: Sorafenib inhibits the shedding of major histocompatibility complex class I-related chain A on hepatocellular carcinoma cells by down-regulating a disintegrin and metalloproteinase 9. Hepatology 51: 1264-1273, 2010.

33. Chotjumlong P,Bolscher JG, Nazmi K, Reutrakul V, Supanchart C, Buranaphatthana W and Krisanaprakornkit S: Involvement of the P2X7 purinergic receptor and c-Jun $\mathrm{N}$-terminal and extracellular signal-regulated kinases in cyclooxygenase-2 and prostaglandin E2 induction by LL-37. J Innate Immun 5: 72-83, 2013.

34. Izumi Y, Hirata M, Hasuwa H, Iwamoto R, Umata T, Miyado K, Tamai Y, Kurisaki T, Sehara-Fujisawa A, Ohno S and Mekada E: A metalloprotease-disintegrin, MDC9/meltrin-gamma/ADAM9 and PKCdelta are involved in TPA-induced ectodomain shedding of membrane-anchored heparin-binding EGF-like growth factor. EMBO J 17: 7260-7272, 1998.

35. Peduto L, Reuter VE, Shaffer DR, Scher HI and Blobel CP: Critical function for ADAM9 in mouse prostate cancer. Cancer Res 65: 9312-9319, 2005.

36. Lindenblatt Rde C, Martinez GL, Silva LE, Faria PS, Camisasca DR and Lourenço Sde Q: Oral squamous cell carcinoma grading systems-analysis of the best survival predictor. J Oral Pathol Med 41: 34-39, 2012.

37. Peduto L: ADAM9 as a potential target molecule in cancer. Curr Pharm Des 15: 2282-2287, 2009. 